\title{
Investigations: how to get from guidelines to protocols
}

\author{
Firstly, collect the right data
}

Often the diagnostic process of interview and investigation entails a complex but routine sequence of decisions. ' Guidelines based on advance analysis of a general form of each problem may therefore simplify the management of individual patients. Abdominal, back, and chest pain; gastrointestinal bleeding; diarrhoea; dysuria; mild dyskaryosis; vaginal discharge; head injury; and epilepsy have all been seen as amenable to this process, ${ }^{2-6}$ and writing guidelines for such routine cases has become fashionable.

Stimulus for this comes from evidence that opinion on diagnosis and management may vary greatly, ${ }^{8}$ with wide, unjustifiable variations in clinical practice. ${ }^{9}$ Differences do not necessarily directly disadvantage the patient, but they form the central difficulty in managing a health system that is, simplistically, just the sum of all individual clinical decisions. Other potentially conflicting pressures have arisen from the requirements to define good practice for clinical audit and cost effective investigation and to define negligent practice for legal purposes.

The Royal College of Pathologists and the Audit Commission have both endorsed guidelines for investigation as a way to control the demand for medical laboratory services, which cost the NHS one third of a billion pounds each year. ${ }^{10}$ The Royal College of Radiologists has drawn up guidelines on the use of 12 common $x$ ray examinations. ${ }^{11}$ The Royal College of Physicians has discussed the use of investigations, ${ }^{12}$ as have the consensus conferences of the King's Fund ${ }^{13}$ and the National Coordinating Network for Cervical Cytology. ${ }^{5}$ At least one academic unit is producing guidelines to bridge primary and secondary care. ${ }^{1+}$ In the United States guidelines and protocols have been controversial. The closely argued Common Diagnostic Tests: Use and Interpretation of the American College of Physicians, derived from detailed information by probabilistic reasoning, has been fiercely criticised for years. ${ }^{15} 16$ Yet guidelines are now enshrined by act of Congress in the new Agency for Health Care Policy and Research. ${ }^{17}$

The best form for guidelines and their effectiveness in practice remain unclear. Most current British guidelines are descriptive accounts of principles of good practice decided in a pragmatic, unstructured way by expert groups supported by reviews of published work with some formal analysis of controlled trials, including meta-analysis. ${ }^{18}$ The group approach, however, has serious flaws. It continues the biases of traditional, empirical, medical practice, evolved from collective personal experience, which is sanctioned by experts. ${ }^{19}$ Decisions are oversimplified, and assessments of risk are based on limited and selected cases. What results is a balance between conflicting views controlled by professional conservatism and pressure for consensus. Whereas a genuine consensus of experts should produce a "best guess," often variation in opinion is too enormous. ${ }^{20} 21$ Analysis of social choice shows that systems of ranking opinion to cope with this-for example, that of the American RAND Corporation ${ }^{122}$-may lead to arbitrary and spurious decisions. ${ }^{22}$ Such weaknesses offer easy targets for the champions of clinical freedom, tempting those writing guidelines to cover all options by adopting imprecision or stating only the lowest common denominator. Alternatively, a single practice may be endorsed when various alternatives may be equally satisfactory.

Subjective guidelines are open to misuse: a minimally acceptable standard set for audit or litigation could be taken as the baseline by a budget conscious management; on the other hand, an ideal approach could be used for litigation. None the less, limited studies have shown that guidelines are acceptable as standards and can be effective in improving clinical practice. ${ }^{23}$ But guidelines may be slow to evolve and short lived, and they must be constantly reinforced as part of a continuing programme of improving quality. ${ }^{24}$ Such direction can be seen as professionally demeaning - a justifiable reaction when guidelines are so arbitrarily constructed. ${ }^{23}$

The complexity of modern diagnostic investigation, which uses multiple tests in varying combinations, demands quantitative analysis to predict accurately an individual patient's risk, which is the basis of diagnosis. ${ }^{25}$ The wide variations in reporting and interpreting clinical information and the results of applying Bayesian statistics to clinical decisions confirm large discrepancies among subjective views of the appropriateness of a test and its quantitative effect on diagnostic probability. ${ }^{18}$

Conventionally the controlled trial has held primacy as the established tool to give the best answer to questions about managing patients. The breadth of problems and possible solutions, the rapid changes in diagnostic techniques, and wide variation in performance and resources among different doctors and centres make its exclusive use unrealistic. ${ }^{26}$ Operational research into clinical practice presents an attractive option. ${ }^{27}$ There are two difficulties: firstly, the right information is not currently collected, and, secondly, its analysis demands suitable methodology and facilities. The information revolution that is slowly gathering pace in the 
NHS could eventually help. Steering information collection away from general management towards clinical management is long overdue, but universal, uncritical, open ended collection of data is no answer.

Doctors, through the professional bodies that have assumed responsibility for guidelines and audit, need to claim ownership of the new information systems urgently. Foresight is needed to identify clinically and epidemiologically important problems and to define the data needed to establish a future generation of guidelines. Selective, active, cooperation is required to obtain relevant data on specific clinical problems from multiple centres. Such data must be validated for reproducibility and interobserver and intraobserver variation, and careful control and matching of cases are essential. Furthermore, studies of the process of diagnosis need supplementing by examination of its effects on outcome and patients' choice. ${ }^{28}$ Eddy claims that there are four stages in developing guidelines: global subjective, evidence based, outcome based, and preference based. But these are really different, concurrent aspects of a continuing process..$^{29}$

Systematic, collaborative collection and analysis of data would allow the creation of precise guidelines (or protocols) with a structured, logical approach to a closely specified clinical problem, employing only appropriate, reproducible data from each case. Such protocols have been proved to be of value in paramedical practice, specifically setting out how to establish a given threshold of diagnostic certainty for a defined problem.

Investigative protocols would give doctors accurate information on the technical performance of medical diagnosis. A portfolio of protocols could be constructed from which the most appropriate could be selected for local use and their performance audited in detail. ${ }^{30}{ }^{31}$ Clinical skill and acumen remain vital to match protocols to local and individual needs and handle cases outside a protocol. ${ }^{32}$ No protocol devised so far achieves absolute diagnostic accuracy, and current failure rates range from $1 \%$ to $10 \% .{ }^{33+4}$ Deciding what level of diagnostic accuracy is acceptable in a particular case is genuinely a matter for individual clinical judgment and the patient's choice.

Doctors should push for research to develop audit beyond regulation and exhortatory, general guidelines. Audit should include specific, precise, efficient, and effective protocols. The present rapid responses to the demands of political expediency should be substituted by a major long term, professional commitment to derive guidelines from the analysis of clinical, operational data.

Director,

D JENKINS

Islington Clinical Research Centre,

Whittington Hospital,

London N195NF

1 Sox HC, Blatt MA, Higgins MC, Marton KI. Medical decision making. Boston: Butterworths, 1987.

2 Komaroff $\mathrm{AL}, \mathrm{W}$ inickoff $\mathrm{RN}$, eds. Common acute illnesses: a problem-oriented textbook with protocols. Boston: Little, Brown, 1977

3 Hopkins A. Clinical features and appropriate investigation in neurological practice. In: Hopkins A, ed. Appropriate investigation and treatment in clinical practice. London: Royal College of Physicians of London, 1989:59-76.

4 Mallison C. Appropriate use of investigations in gastroenterology. In: Hopkins A, ed. Appropriate investigation and treatment in clinical practice. London: Royal College of Physicians of London.

5 Intercollegiate Working Party on Cervical Cytology Screening. Report. London: Royal College of Obstetricians and Gynaecologists, 198

6 Head to head over Harrogate. Head injuries [editorial]. Lancet 1990;335:695-6.

7 Eddy DM. Practice policies - what are they? JAMA 1990;263:877-80.

8 Feinstein AR. A hibliography of publications on observer variability. $\mathcal{F}$ Chronic Dis 1985;38 619-32

9 Chassin MR, Brook RH, Park RE, Keesey J, Fink A, Kosecoff J, et al. Variations in the use of medical and surgical services by the Medicare population. N Engl f Med 1986;314:285-90.

10 Audit Commission. The puthology services. A management review. London: HMSO, 1991.

11 Roval College of Radiologists. Making the best use of a department of radiology: guidelines for doctors. London: Royal College of Radiologists, 1990.

12 Chassin $M$. How do we decide whether an investigation or procedure is appropriate? In: Hopkins A, ed. Appropriate investigation and treatment in clinical practice. London: Royal College of Physicians of London, 1989:21-9.

13 Consensus conference. Treatment of stroke. BMF 1988;297:126-8.

14 Richards T. Islington shares practice guidelines. BMF 1989:299-756-7.

15 Sox $\mathrm{H}$, ed. Common diagnostic tests: use and interpretation. Philadelphia: American College of Physicans, 1987.

Nash DB. Practice guidelines and outcomes. Arch Pathol Lab Med 1990;114:1122-5.

17 Legislated clinical medical [editorial]. Lancet 1990;335:1004-6.

17 Legislated clinical medical [editorial]. Lancet 1990;335:100 18 Lilford R. State of the obstetric art. Luncet 1989;ii:1205-7.

19 Eddy DM. Clinical policies and the quality of clinical practice. N Engl f Med 1982;307:343-7. 19 Eddy DM. Clinical policies and the quality of clinical practice. $N$ Engl f Med $1982 ; 307: 343-7$.
20 Eddy DM. Clinical decision making: from theory to practice. The challenge. $\mathcal{F} A M A$ A 1989;262 3027-30.

21 Brook RH. Practice guidelines and practising medicine. $\mathcal{F} A M A$ 1989;262:3027-30.

22 Restle F. Mathematical models in psychology. Harmondsworth: Penguin, 1971:84-11

23 Lomas J, Anderson (FM, Domnick-Pierre K, Vauda E, Enkin MW, Hannah WJ. Do practice guidelines guide practice? $N$ Engl F Med 1989;321:1306-11.

24 Bareford D, Hayling A. Inappropriate use of laboratory services: long-term combined approach to modify request patterns. BMJ 1990;301:1305-7.

25 Lambird PA. Resource allocation and the cost of quality. Arch Pathol Lab Med 1990;114:1168-72. 26 Birkhead BG, Jackson RRP. Randomised trials: a perspective. In: Slevin ML, Staquet MJ, eds. Randomised trials in cancer: a critical review by site. New York: Raven Press, 1986:665-75

27 Jackson RRP. The evaluation of clinical trials. Postgrad Med f 1985;61:133-9.

28 Greenfield $S$. The state of outcome of research: are we on target? N Engl f Med 1989;320:1142-3.

29 Eddy DM. Clinical decision making: from theory to practice. Practice policies: where do they come from? JAMA 1990;263:1265-75.

30 Greenfield S. Measuring the quality of office practice. In: Goldfield N, Nash DB; eds. Providing quality care. Philadelphia: American College of Physicians, 1989:183-224.

quality care. Philadelphia: American College of Physicians, $1989: 183-224$.
Pringle 1 . The new agenda for general practice computing. BMf 1990;301:827-8.

32 Blois MS. Information and medicine. Berkeley: University of California Press, 1984

33 Heckerling PS, Tape TG, Wigton RS, Kim K. Hisson G, Leikin JB, et al. Clinical prediction rule for pulmonary infiltrates. Ann Intern Med 1990;113:664-70.

34 Epstein PE. Cassandra and the physician: are clinical prediction rules changing the practice of medicine? Ann Intern .Med 1990;113:646-7.
Treating hypertension is an important part of preventive medicine, particularly in general practice, ${ }^{12}$ where knowing a patient's blood pressure has become synonymous with good practice. ${ }^{3}$ Recently, however, doctors have questioned the value of treating certain levels of hypertension, especially when it is an isolated abnormality. Anomalies exist-for example, controlling hypertension does not invariably reduce coronary heart disease, for which it is an important risk factor. The complex relation between hypertension and other risk factors is only now being teased out, ${ }^{+5}$ leading to a reevaluation of values when treatment is justified. ${ }^{\circ}$

Until now patients have been taught that hypertension means treatment for life, although countless thousands of them have abandoned treatment without their doctors' knowledge or consent. Feeling perfectly well, they have made their own judgments about the value of treatment and the acceptability of its side effects. ${ }^{7}$ Knowing what proportion of them have come to grief is impossible without formal follow up studies, but most doctors have come across patients with normal blood pressures who had previously stopped antihypertensive treatment.

This is compatible with the findings of two large studies. In the Australian therapeutic trial nearly half the placebo group had blood pressures below the entrance criteria after three years. In the Medical Research Council's trial of treatment of mild hypertension between one third and one half of the placebo group had diastolic blood pressures below $90 \mathrm{~mm} \mathrm{Hg}$ (phase V) on one of their anniversary visits, and almost one fifth had values below this at the end of three anniversary visits. 